\title{
RECONOCIMIENTO DE FIGURAS GEOMÉTRICAS A TRAVÉS DE LA PROPUESTA DE INSTALACIONES PSICOMOTRICES
}

\section{THE RECOGNITION OF GEOMETRIC FIGURES THROUGH THE PROPOSAL OF PSYCHOMOTRIC FACILITIES}

\section{Nogué-Vila, LI.1ACDF; Marquès- Aregay, L.2ABC}

1 Nogué Vila, LI.; Profesor Departamento de Didácticas Específicas de la Universitat de Girona, España, lluis.nogue@udg.edu

2 Marquès Aregay,L. 2BC; Graduada en Magisterio Educación Infantil, Mención Educación

Física. Docente. España, laura_marques_14@hotmail.com

Responsabilidades

A Diseño de la investigación

в Recolector de datos

c Redactor del trabajo

D Tratamiento estadístico

E Apoyo económico

F Idea original y coordinador de toda la investigación

Recibido el 5 de enero de 2020

Aceptado el 26 de febrero de 2020

Correspondencia: Lluís Nogué Vila. lluis.nogue@udg.edu

DOI: http://dx.doi.org/10.24310/riccafd.2020.v9i1.8308

\section{RESUMEN}

Este proyecto de innovación docente tiene como objetivo estudiar la incidencia de la intervención psicomotriz a través de la propuesta de instalaciones psicomotrices de los autores Abad y Ruizı en el aprendizaje de las formas geométricas. Las instalaciones psicomotrices se basan en la creación y composición de espacios mediante la disposición de objetos estéticamente atractivos. Las instalaciones psicomotrices creadas mantienen una relación geométrica entre objetos y la composición en el espacio. Partiendo de los principios de la Educación Matemática Realista2,3 (EMR) se propuso analizar la posible mejora del reconocimiento de las figuras geométricas des de la intervención psicomotriz de Aucouturier4. En base al método de Investigación Basado en el Diseño, utilizando un diseño preexperimental pretest y posttest, se generaron datos en relación al 
reconocimiento de figuras geométricas según los fundamentos de la EMR. Los resultados muestran una clara mejora en el reconocimiento de las figuras geométricas básicas.

PALABRAS CLAVE: psicomotricidad, transversalidad, arte y matemáticas

\begin{abstract}
This teaching innovation project aims to study the incidence of psychomotor intervention, through the facilities proposed by the authors Abad and Ruiz 1 in the learning of basic geometric shapes. The psychomotor facilities are based on the creation and composition of spaces through the arrangement of aesthetically attractive. The psychomotor facilities shows a geometric relationship between objects and composition in space. Starting from the principles of Realistic Mathematical Education2,3 (MRE) it was proposed to analyze the possible improvement of the recognition of geometric figures from the psychomotor intervention of Aucouturier4. Based on the Design Based Research method, using a pre-experimental pre-test and post-test design, data were generated in relation to the recognition of geometric figures according to the fundamentals of MRE. The results obtained show a clear improvement in the recognition of basic geometric figures.
\end{abstract}

KEY WORDS: psychomotricity, transversal competences, art and maths

\title{
INTRODUCCIÓN
}

Esté proyecto de innovación yace del estudio y del interés suscitado por el modelo de intervención psicomotriz de los autores Abad5,6 y Abad y Ruíz1 a través de su propuesta de instalaciones. El proyecto conllevó la realización de una investigación acción7 con el objetivo de evaluar la incidencia de un modelo de intervención psicomotriz basado en dicha propuesta de instalaciones y el aprendizaje y reconocimiento de las formas geométricas simples.

Abad y Ruíz1 proponen un trabajo de intervención psicomotriz desde la creación de espacios singulares (instalaciones); estéticamente atractivos, de composición espacial estructurada, de sensibilidad cromática y estrechamente vinculados a la concepción de las producciones del arte contemporáneo. La propuesta que defienden Ruiz y Abads considera que la sensibilidad del espacio permite una interacción facilitadora entre el niño/a, el espacio y la producción simbólica. El análisis de algunas de las instalaciones de los autores permite ver que, en la gran mayoría, predominan elementos geométricos, ordenada y conscientemente dispuestos, dibujando al unísono otras formaciones geométricas en el espacio. En este caso se han creado dichas 
instalaciones siguiendo las características descritas por los autores Ruiz y Abad8, permitiéndonos elaborar espacios/instalaciones que mantengan claramente un contenido geométrico concreto e intencionado, hecho que nos permite evaluar el objetivo de nuestro estudio (ver figura 1). Además, consideramos, como manifiestan Edo y Revelles9, Edo11 citado por Antón y Gómez10p100, que el aprendizaje de las figuras geométricas básicas debe ser de manera globalizada y transversal, así como debería permitir el juego del niño/a a través de la visualización, manipulación e interacción con una propuesta de instalación. Al mismo tiempo, esta instalación de carácter artístico permite el desarrollo de competencias geométricas al estar representadas propiedades de forma que permitan la adquisición de formas geométricas básicas12citado por citado por Antón y Gómez10p100.
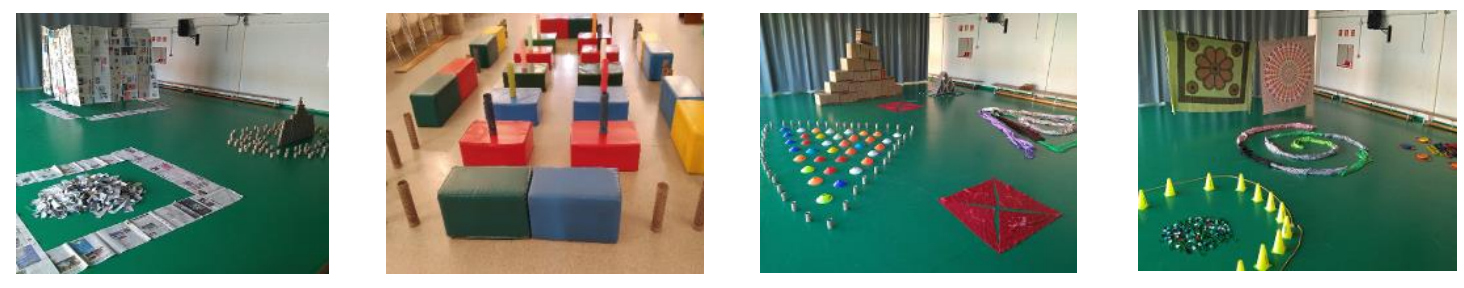

Figura 1: algunas instalaciones con contenido geométrico intencionado

La creación de una instalación requiere de un espacio abierto, diáfano, que facilite una construcción estéticamente insinuante y atrayente, que permita la contemplación inicial del niño/a para la inmediata y consecuente actuación e interacción con el espacio y los materiales partiendo de su movimiento libre, espontaneo y creativo. Será su creatividad motriz y cognitiva que permitirán explorar las posibilidades y características de los materiales, así como nuevas creaciones, siempre desde la interacción de la motricidad gruesa y fina para aflorar sus creaciones simbólicas.

Igual que los autores Ruiz y Abad8 proponemos un modelo de intervención psicomotriz en la línea relacional de Aucouturier4, partiendo de la espontaneidad motriz y favoreciendo el desarrollo integral des del "diálogo tónico" psicomotricista-niño/a, entendiendo por este concepto una relación e intercambio interpersonal corporal, psíquico y emocional como construcción del yo a partir del "diálogo" con el otro13. En este caso, el modelo de intervención mantuvo los principios de la Práctica Psicomotriz d'Aucouturier4 con la diferencia metodológica de incorporar una breve intervención dialógica inicial por parte de la docente para incidir sobre el objetivo de estudio; las formas geométricas.

Como hemos argumentado la propuesta de instalación psicomotriz proyecta espacios estructurados intencionadamente, en nuestro caso organizados con un marcado contenido geométrico con el fin de valorar si a través de su interacción y proyección simbólica favorece el aprendizaje y el reconocimiento de las figuras geométricas. Así, consideramos que el niño/a 
podrá transcurrir del juego simbólico personal al proyectado14 o viceversa a través del espacio, favoreciendo un trabajo de proyección y construcción sobre los objetos y en consecuencia una mejora en el aprendizaje de las características de las figuras geométricas simples. En definitiva, una transformación del mundo exterior mediante el juego para una mejor adecuación y aprendizaje del mundo externo15. Basándonos en los principios de la teoría de la Educación Matemática Realista (EMR) Freudenthal2 (1991), citado por Alsina, Novo, Moreno3 p2, y en las aportaciones de Alsina15 sobre ésta misma perspectiva, consideramos que el aprendizaje toma un máximo sentido des de la práctica de contextos reales ajustados a las realidades de los niños para el aprendizaje de las matemáticas. Así, la geometría, tendrá mayor éxito en su aprendizaje si partimos de contextos reales y manipulativos como pueden ser las instalaciones psicomotrices que defendemos en éste trabajo.

De la observación de sus producciones, del intercambio relacional entre iguales y/o docente y de la proyección motriz simbólica sobre las instalaciones geométricamente intencionadas yace la propuesta de innovación de analizar y evaluar el aprendizaje y reconocimiento de las figuras geométricas simples desde los principios de la EMR. Así, con tal contexto de investigación, se pretende afirmar que la intervención psicomotriz d'Aucouturier, favorece el aprendizaje y el reconocimiento de las formas geométricas básicas a través de las propuestas de instalaciones psicomotrices.

\section{MATERIAL Y MÉTODOS}

Para el estudio se utilizó el método de Investigación Basado en el Diseño (DBR) (en el marco de los llamados métodos orientados a la acción), a partir de un diseño pre experimental pretest y posttest. El instrumento empleado (test) se creó en relación al reconocimiento de figuras geométricas según los fundamentos de la EMR con el fin de evaluar cuantitativamente el reconocimiento de las figura geométricas básicas.

El estudio se realizó en la escuela pública de Gerona, con un grupo natural de P4. Cabe decir que la escuela funciona por grupos heterogéneos de edad, en este caso P3 y P4, pero el estudio solo se ha llevado a cabo con los alumnos de $\mathrm{P} 4$, con una muestra total de 12 alumnos.

El estudio se realizó durante cinco sesiones (impacto). Anteriormente se pasó un pretest a los alumnos participantes del estudio y una vez finalizadas las cinco sesiones de impacto de intervención psicomotriz a partir de instalaciones psicomotrices se volvió a pasar el mismo tests evaluativo (posttest).

El test evaluativo es de propia elaboración y validado por tres expertos en el tema, para valorar y registrar el nivel de reconocimiento de las siguientes figuras geométricas básicas: triangulo, círculo, cuadrado y rectángulo. El test está basado en los principios de la EMR y los postulados del reconocimiento de las figuras geométricas en los contextos reales, "matematización del entorno"16, 
es decir, a partir de imágenes propias de su entorno escolar. El test registra el número de reconocimientos de figuras geométricas y aciertos/errores sobre dos cartulinas; una con fotografías de espacios concretos de la escuela y otra con dibujos de autores expresionistas (Klee, Kandinsky, Mondrian). El hecho de presentar ésta segunda configuración de imágenes se debe a la relación del trabajo a nivel transversal con el arte contemporáneo y el tratamiento de las figuras geométricas.

Cabe recordar que el estudio estaba destinado a alumnos de P4 de Educación Infantil con lo cual el test debía presentar máxima sencillez y efectividad en respuesta a las características psicoevolutivas de la muestra. Limitamos el test a dos preguntas para minimizar el tiempo de atención y facilitar la capacidad de concentración. El test consistía en formalizar una primera pregunta de reconocimiento; "Ves está forma en este dibujo? Si ves alguna señálala con el dedo" sobre la cartulina con dibujo/cuadro artístico de un autor expresionista y una segunda frase; "Ves esta forma en esta fotografía?" sobre una cartulina con fotografías del entorno próximo de la escuela sobre las cuatro figuras geométricas citadas. Se utilizó exclusivamente el lenguaje verbal y la señalización con el dedo por parte del alumno de las figuras reconocidas para evitar injerencias de la lectoescritura, del lenguaje y del cómputo numérico. La verbalización de la pregunta era acompañada con la figura geométrica correspondiente a reconocer, de madera y sin color añadido para facilitar la exploración y observación. La pregunta no nombraba el vocablo de la figura geométrica para evitar así las posibles confusiones entre la palabra, el significante y la figura referenciada. ¿Una vez reconocida la figura geométrica la docente alentaba al alumno que prosiguiera el reconocimiento verbalizando la pregunta "Ves alguna más?". El registro era realizado por la docente en una plantilla con los mismos dibujos y fotografías en blanco y negro sobre el cual se marcaban con una cruz de distinto color la figura reconocida para facilitar las consiguientes discriminaciones geométricas que observaba. Las cartulinas del test respetaban los colores naturales del dibujo o espacio fotografiado para ceñirse a la realidad mientras que la plantilla de registro era en blanco y negro para limitar la posible curiosidad del alumno y así favorecer su atención sobre las cartulinas del test. Posteriormente la docente recogía los aciertos y errores en una tabla de registro con la posibilidad de incluir comentarios o observaciones para un posterior análisis. La sesión era grabada para posibles análisis complementarios.

El test evaluativo inicial, pre impacto, se pasó de manera individual, en una aula exclusiva solo para el alumno y la psicomotricista/docente para evitar distracciones. La docente se basó en crear un clima de confianza a partir del diálogo con el alumno siguiendo la misma metodología que en aula ordinaria. Explicaba brevemente la actividad y se formulaban las preguntas descritas anteriormente para el reconocimiento de las figuras geométricas sobre ya dichas cartulinas. El test pre-impacto se pasó inicialmente, antes de intervenir con la propuesta de psicomotriz de instalaciones. Posteriormente se volvió a pasar la misma prueba manteniendo las mismas condiciones de ejecución.

La intervención se basó en cinco sesiones de 60 minutos. Cada sesión presentaba una instalación diferente, creada conscientemente en relación con 
una forma geométrica concreta. La primera sesión se destinó al triángulo, la segunda al cuadrado, la tercera al rectángulo, la curta al círculo y la última a las cuatro figuras geométricas al unísono (ver figura 2). Cada instalación estaba creada en relación con una figura geométrica, des de la propia figura geométrica bidimensional 0 tridimensional del material como de las construcciones producidas con múltiples materiales, siempre con el objetivo de reforzar el mismo concepto geométrico de la sesión. Cabe decir que en la sesión podría contener algún material con alguna otra figura geométrica de manera intrínseca (ejemplo de un cono: circulo i triángulo) pero siempre de manera secundaria o residual. Cada sesión mantenía la estructura propia de la perspectiva de la práctica psicomotriz de Aucouturier 4 (rituales y espacios) aunque, como se ha descrito en las primeras líneas, con un apunte inicial de una breve introducción verbal del psicomotricista sobre la instalación. Intervención que pretendía favorecer la observación intencionada sobre la instalación a nivel estético y geométrico. Posteriormente los alumnos interactuaban de manera libre y espontánea sobre la instalación con las consecuentes construcciones y proyecciones propias.

\begin{tabular}{|c|c|c|c|c|c|}
\cline { 2 - 6 } \multicolumn{1}{c|}{} & SESIÓN 1 & SESIÓN 2 & SESIÓN 3 & SESIÓN 4 & SESIÓN 5 \\
\hline $\begin{array}{c}\text { Ritual } \\
\text { Entrada }\end{array}$ & $\begin{array}{c}\text { Diálogo, } \\
\text { conceptos y } \\
\text { soporte visual }\end{array}$ & $\begin{array}{c}\text { Diálogo, } \\
\text { conceptos y } \\
\text { soporte visual }\end{array}$ & $\begin{array}{c}\text { Diálogo, } \\
\text { conceptos y } \\
\text { soporte visual }\end{array}$ & $\begin{array}{c}\text { Diálogo, } \\
\text { conceptos y } \\
\text { soporte visual }\end{array}$ & $\begin{array}{c}\text { Diálogo, } \\
\text { conceptos y } \\
\text { soporte visual }\end{array}$ \\
\hline $\begin{array}{c}\text { Parte } \\
\text { Principal }\end{array}$ & $\begin{array}{c}\text { Instalaciones } \\
\text { Instalaciones }\end{array}$ & Instalaciones & & \\
\hline $\begin{array}{c}\text { Ritual } \\
\text { Salida }\end{array}$ & Dibujo & $\begin{array}{c}\text { Relacionar } \\
\text { cualidades }\end{array}$ & Dibujo & $\begin{array}{c}\text { Relacionar } \\
\text { cualidades }\end{array}$ & Dibujo \\
\hline
\end{tabular}

Figura 2: figuras geométricas trabajadas en cada sesión

Finalizadas las cinco sesiones de impacto se volvió a pasar el test evaluativo, post impacto, para un posterior análisis de los datos recogidos.

\section{RESULTADOS}

El análisis de los datos recogidos se trataron de manera cuantitativa a partir de los resultados del test pre-impacto y post impacto y la correspondiente comparativa entre ellos tanto a nivel individual como en grupo.

El test permite reconocer un total de 26 triángulos, 40 cuadrados, 70 rectángulos y 20 círculos. Atendiendo que el número de formas geométricas no es igual en cada forma la expresión de los valores se hizo en porcentajes fara así facilitar la comparativa entre las diferentes formas.

El análisis individual muestra el valor de aciertos de pre impacto, de post impacto y el porcentaje de mejora de cada forma geométrica. Los 
resultados individuales muestran una mejora en cada una de las formas geométricas. Así en el caso del triángulo los alumnos mayoritariamente mejoran con valores entre el $13 \%$ i el $38 \%$, al que cabe destacar un caso de mejora del $62 \%$, un caso con una mejora de solo el $3 \%$, otro manteniendo el mismo valor inicial y un solo caso de desmejora con un -3\%. En relación con el cuadrado todos los alumnos han presentado una mejora en el reconocimiento de las figuras geométricas en relación con valores del $10 \%$ al $55 \%$ exceptuando dos casos con mejoras mínimas del $4 \%$ y $5 \%$ y otro sin mostrando el mismo valor inicial. Los resultados en relación con el rectángulo muestran una mejora en todos los casos con valores van del $25 \%$ al $72 \%$. En el caso del círculo también muestran una mejora prácticamente todos los alumnos con valores entre el $10 \%$ y el $65 \%$ exceptuando un alumno que presenta el mismo valor inicial y otro con una desmejora del $5 \%$. Cabe decir que los alumnos que presentan valores de mejora bajos, idénticos o de desmejora, es decir entorno al 5\%, 0\% o inferior a éste último valor son pocos y en ningún caso corresponden al mismo alumno.

El análisis de los resultados a nivel global nos permite una visión más genérica de cada forma geométrica y como no del objetivo del estudio. Los resultados globales reafirman que ha habido una mejora en el reconocimiento de todas y cada una de las figuras geométricas con valores superiores al $20 \%$ y en un caso muy superior. Así, el triangulo, a nivel grupal muestra una mejora del $21,80 \%$, el cuadrado del $26,70 \%$, el círculo del $25 \%$ y el rectángulo un valor de mejora del 47,70\%. (Ver figura 2).

\section{VALORES DE LA MUESTRA}

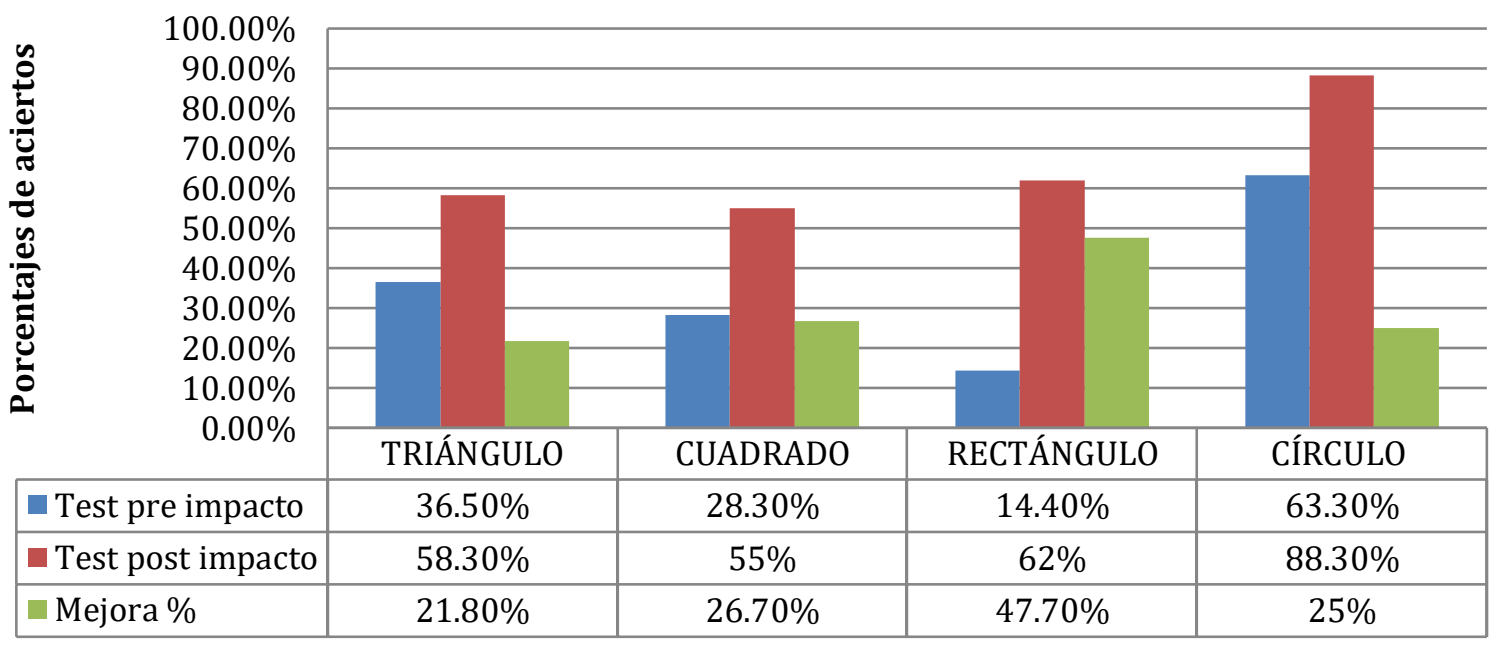

Figura 3: análisis comparativo global en porcentajes de la evolución de aciertos en relación al test pre impacto y post impacto.

\section{DISCUSIÓN}

Los resultados concluyen que la intervención psicomotriz en espacios de juego contextualizados e organizados según las características de las 
propuestas de instalaciones psicomotrices 1,8 , respetando un sentido estético, harmónico y geométrico, permiten una interacción del alumno con los principios del aprendizaje de la matemática realista3 y de "matematización del entorno"16 favoreciendo así una aprendizaje de las figuras geométricas simples.

Mayoritariamente la literatura científica se ha centrado en el estudio correlativo del desarrollo psicomotor y el desarrollo de las competencias matemáticas 0 el pensamiento lógico-matemático17,18. La investigación presentada se centra en una propuesta de intervención metodológica contextualizada y no propiamente en el desarrollo de capacidades psicomotrices ya que parece más que evidenciado. Consideramos que el estudio se alinea con el estudio de López Rodriguez17 ,o mejor dicho una prolongación, en relación a la exploración activa del espacio y de los espacios tridimensionales en la sala de psicomotricidad en el ciclo de 0 a 3 años. López Rodriguez19, a partir de diversas situaciones observacionales, correlaciona la exploración del espacio a partir del juego y la aproximación a contenidos matemáticos. La propuesta de instalaciones psicomotrices 8 es, en definitiva, una organización intencionada del espacio sobre el cual se interacciona des del juego libre y espontaneo y la intervención del docente orientada a la adquisición y reconocimiento de figura geométricas en un ciclo educativo superior.

Con lo cual se evidencia que una actividad motriz, acompañada de una intervención pedagógica docente adecuada, puede ser un elemento importante en el proceso de aprendizaje a partir de la interacción con el entorno. Así, podríamos considerar que el juego motriz, en contextos favorecedores de aprendizaje y con una propuesta metodológica ajustada a los objetivos educativos, puede ser una sustancial herramienta pedagógica para el desarrollo integral del alumno.

No cabe duda que la actividad física conlleva importantes resortes en el campo educativo que van más allá de la dimensión motriz. Resortes sobre los cuales los profesionales de la actividad física debemos saber transmitir y divulgar para darle la importancia que se merece.

\section{LIMITACIONES Y LÍNEAS FUTURAS}

La presente investigación presenta unas limitaciones que se deben de declarar. Los resultados están limitados por el diseño de las actividades mismas. Sería necesario formular mayor numero de situaciones de aprendizaje y a un mayor número de alumnos. Los valores de la muestra sin duda no permiten generalizaciones. Se precisan, por lo tanto de estudios que incorporen información longitudinal sobre la efectividad, así como la relación directa con la consecución de competencias matemáticas con la intervención psicomotriz en contextos de instalaciones psicomotrices según los criterios aquí propuestos. 


\section{REFERENCIAS BIBLIOGRÁFICAS}

1. Abad J, Ruiz A. Contexto de simbolización y juego: La propuesta de las instalaciones. Revista Aula de Infantil. 2014;77,11-15.

2. Freudenthal H. Revisiting mathematics education. Dordrectht: Kluwer Academic Publishers. 1991.

3. Alsina A, Novo M, Moreno A. Redescubriendo el entorno con ojos matemáticos: Aprendizaje realista de la geometría en Educación Infantil. Edma 0-6: Educación Matemática en la Infancia. 2016; 5(1),1-20.

4. Aucouturier B. Los fantasmas de acción y la práctica psicomotriz.7a ed. Barcelona: Editorial Graó. 2015.

5. Abad J. Compromiso del artista contemporáneo en contextos educativos: El artista residente en la escuela como colaborador del proyecto estético. 2014. Manuscrito inédito, Universidad Autónoma de Madrid.

6. Abad J. Configuración del espacio en la escuela infantil: La escuela como ámbito estético según la pedagogia Reggiana. 2012. Manuscrito inédito. Universidad de Madrid (CSEU La Salle).

7. Latorre A. La Investigación-Acción: Conocer y cambiar la práctica educativa. Barcelona: Editorial Graó, 2008.

8. Ruiz A, Abad J. El juego simbólico. 7a ed. Barcelona: Editorial Graó, 2018.

9. Edo, M. y Revelles, S. "Situaciones matemáticas potencialmente significativas" a M. Antón C. y B. Moll (coords.). Educación Infantil. Orientaciones y Recursos (0-6 años). 2004. CISSPRAXIS. Barcelona. pp.410/103-410/179

10. Antón A, Gómez M. La geometría a través del arte en educación infantil. Enseñanza \& teaching.2016;34,94-117. Disponible en: https://revistas.usal.es/index.php/0212-374/article/view/et201634193117/15157 Doi: 10.14201/et201634193117.

11. Edo M. Matemáticas y arte en la Educación Infantil. Uno: 2008;47: 3753.

12. Vallejo López, F. Las matemáticas en el arte: su didáctica. Revista Digital Ciencia y Didáctica. 2011; 50: 73-83.

13. Franch N. Poètica Corporal. Barcelona: Ediciones Octaedro, 2018.

14. Slade P. Expresión dramática infantil. Madrid: Santillana Texto Editorial, 1988.

15. Bruner J. Acción, pensamiento y lenguaje. Madrid: Alianza Editorial,1984.

16. Alsina A. La "pirámide de la educación matemática": una herramienta para ayudar a desarrollar la competencia matemática. Aula Innovación Educativa. 2010;189:12-16.

17. Noguera Machacón LM, Herazo Beltrán Y, Vidarte Claros JA. Correlación entre perfil psicomotor y rendimiento lógico-matemático en niños de 4 a 8 años. Rev Cienc Salud 2013; 11 (2): 185-194.

18. Gómez S. Influencia de la motricidad en la competencia matemática básica en niños de 3 y 4 años Edma 0-6: Educación Matemática en la Infancia (2014) 3(1), 49-73.

19. López Rodríguez M. Matemáticas y psicomotricidad de 0 a 3 años. Edma 0-6: Educación Matemática en la Infancia. 2015; 4(1),43-47. 